\title{
Automatic Extraction of Planetary Image Features
}

\author{
G. Troglio, J. Le Moigne, G. Moser, S. B. Serpico, J. A. Benediktsson,
}

\begin{abstract}
With the launch of several Lunar missions such as the Lunar Reconnaissance Orbiter (LRO) and Chandrayaan-1, a large amount of Lunar images will be acquired and will need to be analyzed. Although many automatic feature extraction methods have been proposed and utilized for Earth remote sensing images, these methods are not always applicable to Lunar data that often present low contrast and uneven illumination characteristics. In this paper, we propose a new method for the extraction of Lunar features (that can be generalized to other planetary images), based on the combination of several image processing techniques, a watershed segmentation and the generalized Hough Transform. This feature extraction has many applications, among which image registration.
\end{abstract}

Index Terms - Feature extraction, image processing, image registration, image segmentation.

\section{INTRODUCTION}

$\mathrm{T}$ he Lunar Reconnaissance Orbiter (LRO) is a NASA mission, aimed at creating a comprehensive atlas of the moon features and resources to aid in the design of a lunar outpost and to prepare exploration and scientific missions to the Moon. LRO will be launched in late 2008 and will spend at least one year in orbit collecting detailed information about the moon and its environment. Different types of data will be collected by LRO at different times, by different sensors, and from different view-points. Registration will be an essential task to jointly exploit, integrate, or compare these different data, and feature extraction is the first step to not only image registration but any further analysis of these data. Because planetary images typically exhibit lack of contrast, poor illumination and lack of good features, we propose a novel region-based approach for the extraction of Lunar (and planetary) features.

\section{APPROACH}

The features to be extracted are rocks (i.e., objects of small elliptical shape), craters (objects of elliptical shape with shadows), and ridges. In order to detect them, the image gradient is first computed by using the Canny edge detector[1]. Then, the watershed algorithm in [2] is applied to the Canny gradient, in order to segment regions in the gradient image, which appear as closed-contours in the gradient are segmented. Elliptical shapes are detected by a generalized

This work was supported by the Research of Fund of the University of Iceland and was performed at NASA Goddard Space Flight Center.

G. T. and J. A. B. Authors are with the Univ. of Iceland, Reykjavik, 107 Iceland (+3901921945135; giulia.troglio@unige.it and benedikt@hi.is).

G. T., G. M. and S. B. S. Authors are with the University of Genoa, Genoa, 16100 Italy (e-mail: sebastiano.serpico@unige.it).

J. Le M. Author is with the Goddard Flight Space Center, Code 580, NASA, Greenbelt, MD 20770 USA (Jacqueline.LeMoigne@nasa.gov).
Hough accumulator [3]. Furthermore, a standard Hough accumulator [4] can be applied to detect straight lines in the gradient image.

For the application of this method to registration, the features above are extracted from both images to be registered, and then matched in order to compute the geometric transformation required to achieve the registration.

\section{RESULTS}

Since, currently, LRO images are not yet available. experiments were carried out using similar data, collected during the mission Mars Global Surveyor. Preliminary results are shown in Fig.1.

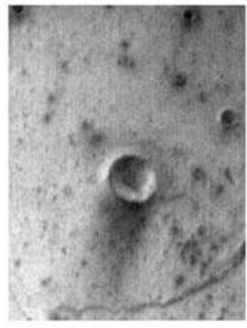

(a)

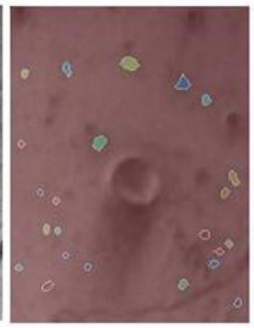

(b)

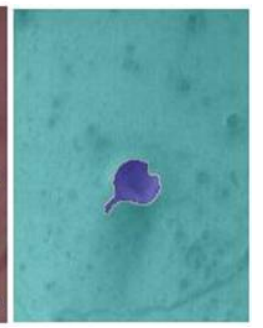

(c)
Fig. 1. The original image (a), the close contour features (b) and the elliptic shape features (c) are shown.

\section{BENEFITS}

The proposed approach will be used for many applications dealing with all the different data collected during the LRO mission (and other Lunar missions), among which image registration and image analysis, with the aim of selecting safe landing sites, identifying lunar resources, and studying how the lunar radiation environment will affect humans.

\section{CONCLUSION}

A novel approach has been proposed for feature extraction and matching in registration of planetary data. In planetary data, the features to be extracted are not as well-contrasted nor -defined as for Earth data. However, small rocks, which are not affected by uneven illumination, can easily be detected. An illumination correction will be necessary to detect all the craters, ridges and big rocks, which have shadows.

\section{REFERENCES}

[1] J. Canny, "A Computational Approach to Edge Detection," IEEE-Trans.Pattern Anlaysis \& Machine Intelligence, 1986.

[2] S. Beucher, "The Watershed Transformation applied to Image Segmentation,” Scanning Microscopy International, 1992.

[3] S. Tsuji, F. Matsumoto, "Detection of Ellipses by a Modified Hough Transformation,” IEEE Trans. on Computers, 1978. 
[4] R. Duda and P. Hart, "Use of the Hough Transform to Detect Lines and Curves in Pictures," Communications of the Association for Computing Machinery (15), 1972. 


\title{
Automatic Extraction of Planetary Image Features
}

\author{
G. Troglio, J. Le Moigne, J. A. Benediktsson, G. Moser, S. B. Serpico
}

\author{
G. Troglio and J. A. \\ Benediktsson \\ Dept. of Electrical and Computer \\ Engineering \\ University of Iceland \\ Reykjavik, Iceland \\ benedikt@hi.is
}

\author{
G. Troglio, G. Moser, and S. \\ B. Serpico \\ Dept. of Biophysics and Electronic \\ Engineering, \\ University of Genoa \\ Genoa, Italy \\ sebastiano.serpico@unige.it
}

\author{
J. Le Moigne \\ Goddard Space Flight Center, \\ Software Engineering Division, \\ NASA GSFC Code 580, \\ Greenbelt MD, USA \\ Jacqueline.LeMoigne@nasa.gov
}

\begin{abstract}
With the launch of several Lunar missions such as the Lunar Reconnaissance Orbiter (LRO) and Chandrayaan-1, a large amount of Lunar images will be acquired and will need to be analyzed. Although many automatic feature extraction methods have been proposed and utilized for Earth remote sensing images, these methods are not always applicable to Lunar data that often present low contrast and uneven illumination characteristics. In this paper, we propose a new method for the extraction of features from the Lunar surface, based on the combination of several image processing techniques, including a watershed segmentation and the generalized Hough Transform. The method has many applications, among which image registration, and can be generalized to other planetary images as well.
\end{abstract}

Crater detection; feature extraction; image segmentation; image registration.

\section{INTRODUCTION}

The Lunar Reconnaissance Orbiter (LRO) is a NASA mission, aimed at creating a comprehensive atlas of the Moon features and resources to aid in the design of a lunar outpost and to prepare exploration and scientific missions to the Moon [1]. Since the Moon is the natural place to test new exploration technology, this return to the Moon will open the way to further space exploration, thus providing a "gateway to the universe".

LRO will be launched in the summer of 2009 and will spend at least one year in orbit collecting detailed information about the Moon and its environment. The spacecraft will be placed in low polar orbit $(50 \mathrm{~km})$ for a 1year mission under NASA's Exploration Systems Mission Directorate. Different types of data (e.g., day-night temperature maps, a global geodetic grid, high resolution color imaging UV albedo) will be collected by LRO at different times, by different sensors, and from different viewpoints. Therefore, registration will be an essential task to jointly exploit, integrate, or compare these different data, and feature extraction is the first step to not only image registration but also any further analysis of these data.
The identification of the features that are present on the planetary surface by a human expert is a time-consuming endeavor. Therefore, a trustworthy automatic procedure to detect the position, structure, and dimension of each feature is highly desirable.

This is a difficult task because limited data are available, the quality of the images is generally low (i.e., it depends on illumination, surface properties and atmospheric state), and the features that are present in the images can be barely visible due to erosion. These features also exhibit different structures and variable sizes.

Among typical features in Lunar- and planet-surface imagery, craters play a primary role. The crater detection problem has been widely addressed and different approaches have been proposed in the literature. The image-based approaches for crater detection can be divided into two main categories: supervised and unsupervised. The supervised methods require the input of an expert and generally use machine learning concepts to train the algorithm to feature extraction. The unsupervised methods are completely automatic and are generally based on pattern recognition techniques. Different approaches have been proposed, based on template matching [2], texture analysis [3], neural networks [4], or a combination of these techniques [5-6].

So far, the detection of the other types of planetary features has not been addressed in the literature.

Because planetary images typically exhibit lack of contrast, poor illumination and lack of good features, we propose a novel region-based approach for the extraction of Lunar (and planetary) features. The proposed approach, aimed at extracting planetary features, is applied to an image registration process, as described in Sec. II. The presentation and analysis of the results is included in Sec. III. Finally, conclusions and ideas for future improvements are presented in Sec. IV.

\section{APPROACH}

Planetary images show the surface of the analyzed planet and its structures.

The aim of this study is to automatically detect the structures that are present in the represented planetary 
surface by using image analysis techniques and use the extracted features to register multitemporal, multisensor, and stereo-images. Given a pair of images to be registered (i.e., an input and a reference images), their features can be extracted and used to estimate the optimum transformation. Once the transformation parameters are assessed, they can be used to register the input image with respect to the reference one. The flowchart of the proposed technique is shown in Figure 1.

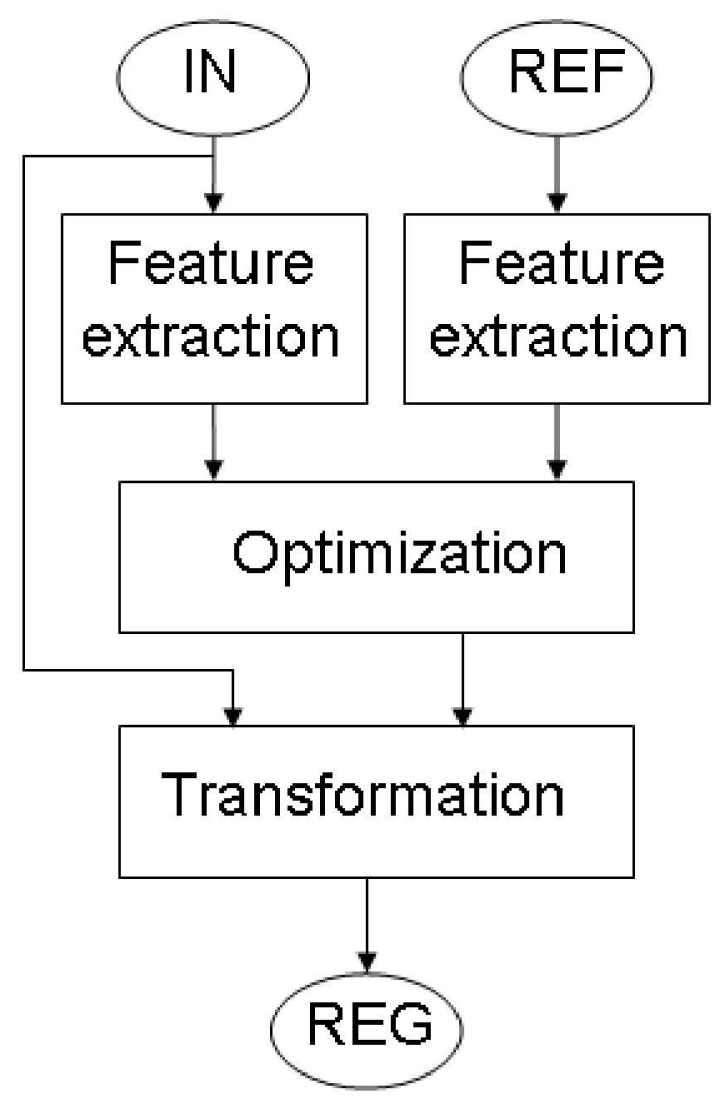

Figure 1. Flowchart of the proposed approach to registration. Features are extracted from both the input and the reference images (IN and REF, respectively). The extracted features are used in an optimization module, aimed at computing the optimum transformation. Once the transformation parameters are estimated, they can be used to transform IN with respect to REF and generate the registered image, REG.

Different types of features are present in the planetary images, and their size, shape and position are estimated by applying different methods, which are presented in Sec. II.A. Once the features are extracted they can be applied to register image pairs, representing the same scene at different observation times. Here, a genetic algorithm is used to optimize the transformation between the pair of images to be registered (see Sec. II.B).

\section{A. Feature Extraction}

The features to be extracted are rocks, craters, and ridges. Rocks are objects of small elliptical or circular shape, with no shadows, craters have approximately elliptical shape with shadows, due to their depth and uneven illumination, and ridges appear like curves and straight lines in the images. Their extraction is a difficult task, because planetary images are blurry, quite noisy, present lack of contrast and uneven illumination, and the represented objects are not well defined. A region-based approach, based on segmentation, has been chosen in order to address such problem. Segmentation is the process of partitioning an image into multiple regions, for instance, in order to distinguish objects from the background. A frequent approach to segmentation introduces a set of characteristic points that are related to the objects to be detected, automatically selected and used as "seed points" to segment the images. Many approaches to segmentation have been explored in the literature. Here, the watershed algorithm in [7] has been chosen, being an automatic, robust and fast method.

Before applying any feature extraction, the images need to be preprocessed. First, the noise is smoothed by applying a Gaussian filtering and a median filtering operation in cascade [8]. Then, in order to detect the edges, the image gradient is computed by using the Canny edge detector [9]. As an intermediate result of this operation an intensity gradient $I_{g}$ is generated, which is a gray-scale image; then, by applying a non-maximum suppression algorithm followed by an hysteresis thresholding to $I_{g}$, a binary gradient image, $I_{b}$, showing the contours of the objects represented in the original image, is obtained.

In order to extract the rocks, which appear like close contours in the gradient image, the watershed algorithm is applied to $I_{b}$, in order to segment regions with close contours. All the areas included within a close contour correspond to "seed point- areas", and are identified as regions. The result of this first step is a binary image that shows the rock boundaries.

While rocks appear like close contours and can be easily detected, craters have a more complex structure and, due to their depth and uneven illumination, exhibit shadows. Their borders can be approximated with incomplete noncontinuous elliptical curves. A generalized Hough accumulator [10] is used to identify the seed points to detect these structures from $I_{b}$. For every pair of points that are detected as edge points in $I_{b}$ and exhibit opposite gradient directions, an accumulator, corresponding to the median point between them, is incremented of a unit value. The maxima of the accumulator are taken as centers of the ellipses. The parameters describing the ellipse centered in each detected maximum are, then, computed; a 3D accumulator is used to estimate the two semi-axes and the direction angle of the ellipse from all the pairs of points that contributed to the accumulator in the considered center. If the ellipse that has been generated truly corresponds to a contour in the gradient image, its center is used as a seed point for segmentation. Starting from all the detected seed points, a watershed algorithm is applied to $I_{g}$ and the craters are identified. As a result, a binary image that shows the crater boundaries is obtained.

Furthermore, a standard Hough accumulator [11] can be applied to detect straight lines in $I_{g}$. Ridges are detected as union of short linear segments. 
A binary image $C$, which represents the contours of all detected features, is created and it is used in the registration process.

\section{B. Registration}

For registration purposes, the features above are extracted from the pair of images to be registered, $I_{i n}$ and $I_{r e f}$, and mapped into two binary images, $C_{i n}$ and $C_{r e f}$, respectively. Such binary images are, then, matched in order to compute the geometric transformation required to achieve the registration.

The proposed registration scheme is based on a global optimization technique aimed at estimating the optimum parameters of an image transformation model. The binary images, which represent the features of $I_{\text {in }}$ and $I_{\text {reff }}$, are fed as inputs to an optimization module. The transformation matrix has to be optimized: its goodness is evaluated by an objective function and its optimization is achieved by applying a genetic algorithm. After the optimum matrix has been estimated it is applied to one of the two images, which is translated and interpolated in order to obtain the final registered image.

The problem is formulated as determining a transformation $T^{*}$ such that, when $T^{*}$ is applied to the first image, $C_{i n}$, the best match with the second one, $C_{r e f}$ is achieved. The objective function to be maximized is:

$$
\operatorname{MOM}(T)=\frac{1}{n} \sum_{(x, y) ; I_{s}(x, y) \neq 0} I_{A}(T(x, y))
$$

where $M O M$ (measure of match) denotes the objective function, $T$ is the transformation for the $x$ and $y$ coordinates in the image plane, and $n$ is the number of nonzero pixels of $C_{\text {ref. }}$ An affine transformation model, which exhibits six independent parameters, is employed.

The determination of the transformation parameters strongly depends on the objective function, as well as on the planetary images to be registered. In this case, where the function has multiple extremes, the most attractive search methods are based on global optimization techniques. In this work, a genetic algorithm (GA) is adopted [12], since it ensures, under mild assumptions, convergence to a global maximum of the adopted matching functional. The six independent parameters of $T$ are defined over a wide range of values to achieve robustness. The aim of the GA is to find the value for such parameters, which maximize the objective function. Given this, the final transformation matrix is calculated, by decoding the fittest individual of the last population and the input image is registered.

\section{RESUlts}

Since currently LRO images are not yet available, experiments were carried out using similar data, which are being collected during the Mars Odyssey mission, by the Thermal Emission Imaging System (THEMIS), an instrument that is on board the Mars Odyssey spacecraft. Such an instrument combines 5-wavelength visual imaging system with a 10-wavelength infrared imaging system [13].
Both 5-bands visible THEMIS images, with a resolution of 18 meters per pixel, and 10-bands infrared THEMIS images, with a resolution of 100 meters per pixel, were used to test the proposed approach.

Results of the feature extraction are shown for a partition of the fourth band of an infrared THEMIS image (Figure 2a).

The image is first preprocessed by a smoothing filter, in order to reduce the noise. The Canny gradient is applied to the smoothed image and results are shown in Figure 2-b. Subsequently, a watershed algorithm is applied to the Canny gradient in order the extract the rocks. Segmentation results and the extracted rock boundaries are shown in Figure 2-c and 2-d, respectively. Finally, the generalized Hough transform is computed and a watershed segmentation is applied, starting the flooding process from the ellipse centers and leading to the detection of the craters. The segmentation result and the crater boundaries are shown in Figures 2-e and $2-f$, respectively.

To demonstrate the applicability of the proposed method to registration, two different non-registered bands of an infrared image are used. In order to show the results the same partition of Figure 2-a is used; in particular, the fourth and fifth bands were selected (Figures 3-a and 3-b, respectively). For both gray-level images, craters and rocks are detected and their contours are extracted and represented in binary contour images, as shown in Figures 3-c and 3-d, respectively. The rotation and translation between the two different bands are visible by looking at Figure 3-e, in which the two non-registered contour images are superimposed in a false-color representation. The contours extracted from the fourth band image are represented in green, whereas the fifth-band contours are shown in red. Finally, the transformation parameters are estimated by the proposed method and the co-registered images are shown in Figure 3f, by using a checkerboard filter. The registration accuracy can be evaluated by looking at the continuity of the image features at the borders of the checks. The visual analysis of the Figure 3-f suggests that the registration performed well; craters and ridges appear continuous at the check borders, i.e., the points of overlap.

\section{CONCLUSION}

A novel approach has been proposed for feature extraction and matching as applied to the registration of planetary data. For such data, the features to be extracted are not as well contrasted nor defined as for Earth data. However, small rocks, which are not affected by uneven illumination, can easily be detected. Crater detection is more critical, because of their depth and spatial extent and consequently their contours are often blurry and not continuous. Nevertheless, we showed in this paper that their identification can be achieved. Moreover, we showed that the extracted features can be used to accurately register pairs of images acquired from the same sensor.

The proposed approach represents the first important step for many applications dealing with all the various data that will be collected during the LRO mission (and other current 
and future Lunar missions), among which image registration and image analysis, with the aim of selecting safe landing sites, identifying lunar resources, and preparing for subsequent Lunar exploration by humans and robots.

In our future work we will investigate the use of an illumination correction algorithm to improve the reliability of the detection for all craters, ridges and large rocks, when shadows are present. Other approaches to locate craters, based on pattern recognition techniques, will also be explored and developed.

Once the feature extraction will be optimized, the approach will be extended to the registration of multisensor images.

\section{ACKNOWLEDGMENT}

This work was supported by the Research of Fund of the University of Iceland and was performed at NASA Goddard Space Flight Center.

\section{REFERENCES}

[1] "Lunar reconnaissance Orbiter", NASA Facts. Goddard Space Flight Center, National Aeronautics and Space Administration, http://lro.gsfc.nasa.gov/mission.html.

[2] A. Flores-Méndez, "Crater marking and classification using computer vision," in Progress in Pattern Recognition, Speech and Image Analysis, vol. 2905, Lecture Notes in Computer Science. New York: Springer-Verlag, 2003, pp. 79-86.

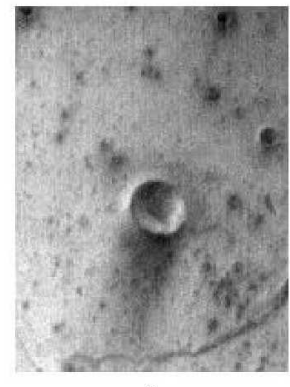

a
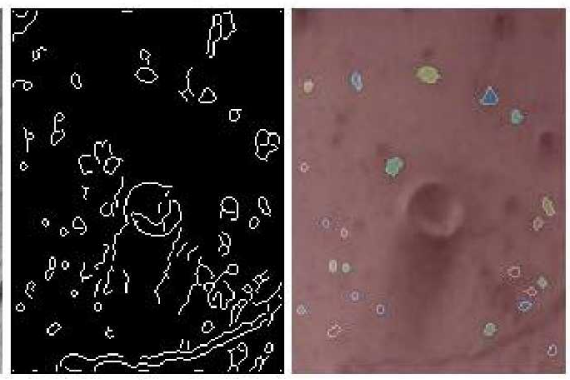

c
[3] J. R. Kim, J.-P. Muller, S. van Gasselt, J. G. Morley, and G. Neukum, "Automated crater detection, a new tool for Mars cartography and chronology," Photogramm. Eng. Remote Sensing, vol. 71, no. 10, pp. $13-22,2000$.

[4] A. A. Smirnov, "Exploratory study of automated crater detection algorithm," Tech. Rep. of Boulder, CO, 2002.

[5] Y. Sawabe, T. Matsunaga, and S. Rokugawa, "Automated detection and classification of lunar craters using multiple approaches," $A d v$. Space Res., vol.37, no. 1, pp. 21-27, 2006.

[6] J. Earl, A. Chicarro, C. Koeberl, P. G. Marchetti, and M. Milsen, "Automatic recognition of crater-like structures in terrestrial and planetary images," in Proc. Lunar Planetary Sci. XXXVI, Houston, TX, 2005, Abs. No. 1319.

[7] S. Beucher, "The Watershed Transformation applied to Image Segmentation," Scanning Microscopy International, 6, 1992.

[8] Shapiro, L. G. \& Stockman, G. C: "Computer Vision", Prentince Hall, 2001

[9] J. Canny, "A Computational Approach to Edge Detection," IEEETrans.Pattern Analysis \& Machine Intelligence, 10(6), 1986.

[10] S. Tsuji, F. Matsumoto, "Detection of Ellipses by a Modified Hough Transformation," IEEE Trans. on Computers, 27(8), 1978.

[11] R. Duda and P. Hart, "Use of the Hough Transform to Detect Lines and Curves in Pictures," Communications of the Association for Computing Machinery, 15, 1972.

[12] Z. Michalewicz, "Genetic Algorithms + Data Structures = Evolution Programs," Springer Verlag, Berlin Heidelberg, third edition, 1999.

[13] P. Christensen, B. M. Jakosky, H. H. Kieffer, M. C. Malin, H. Y. Mesween, K. Nealson, G. L. Mehall, S. H. Silverman, S. Ferry, M. Caplinger, and, M. Ravine. "The thermal emission imaging system (THEMIS) for the Mars 2001 Odyssey Mission". Space Sciece Reviews 100: 85-130,2004.
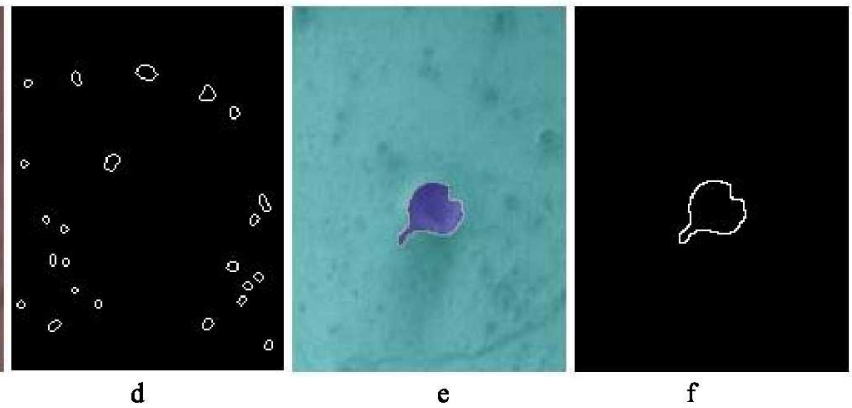

Figure 2. Results are shown for a partition of a THEMIS infrared image (a). The Canny Gradient (b) is segmented (c) and close contours are extracted (d). The Hough accumulator is computed, watershed is applied starting the flooding process from ist maxima (e), and large crater boundaries are, finally, identified ( $f$. 


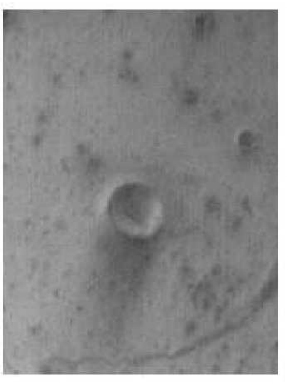

a

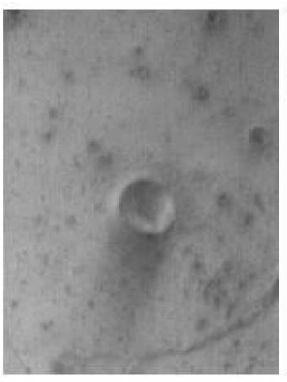

b

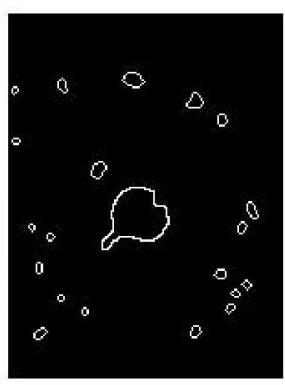

c

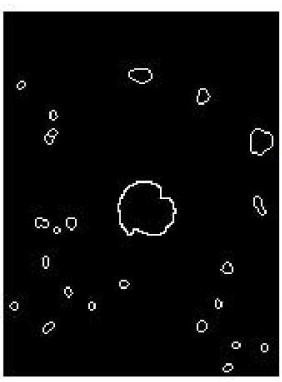

d

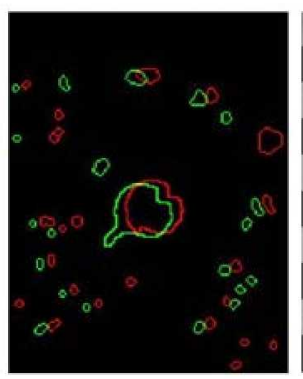

e

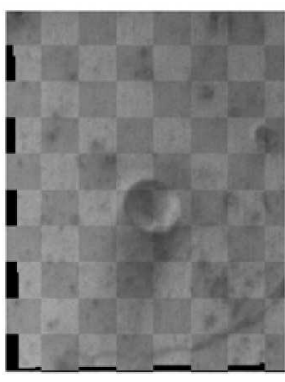

f

Figure 3. Registration results are shown for a partition of the fourth and the fifth bands of a pair of THEMIS infrared images, considered as input (a) and reference image (b), respectively. The gradient of both images are segmented and crater and rock contours are extracted, as shown in (c) and (d),

respectively. The contour images are superimposed and represented in a false-color composition (e), where the green plane is (c) and the red plane is (d), and the blue plane is identically zero. Registration results are shown in (f), by using a checkerboard filter: each check of the board represents the registered input image and the reference image, alternately. 